\title{
Formulation of temozolomide-loaded nanoparticles and their targeting potential to melanoma cells
}

\author{
GUAN JIANG $^{1 *}$, RONGHUA LI $^{1 *}$, JIANQIN TANG $^{1 *}$, YAFENG MA $^{1}$, XIAOYANG HOU ${ }^{1}$, \\ CHUNSHENG YANG ${ }^{2}$, WENWEN GUO $^{3}$, YONG XIN $^{3}$ and YANQUN LIU ${ }^{1}$
}

\author{
${ }^{1}$ Department of Dermatology, Affiliated Hospital of Xuzhou Medical College, Xuzhou, Jiangsu 221002; \\ ${ }^{2}$ Department of Dermatology, Affiliated Huai'an Hospital of Xuzhou Medical College, Huaian, Jiangsu 223002; \\ ${ }^{3}$ Department of Radiotherapy, Affiliated Hospital of Xuzhou Medical College, Xuzhou, Jiangsu 221002, P.R. China
}

Received May 5, 2016; Accepted June 17, 2016

DOI: $10.3892 /$ or.2016.5342

\begin{abstract}
The present study was carried out to prepare and evaluate a temozolomide (TMZ)-loaded polyamide-amine dendrimer (PAMAM)-based nanodrug delivery system, and to explore its ability to target human melanoma (A375) cells in vitro. Firstly, PAMAM-PEG and PAMAM-PEG-GE11 were synthesized by substitution and addition reactions, and their products were identified and characterized by fourier transform-infrared (FTIR), proton nuclear magnetic resonance ( $\left.{ }^{1} \mathrm{H}-\mathrm{NMR}\right)$ and transmission electron microscopy (TEM), as well as differential light scattering (DLS). Using fluorescein isothiocyanate (FITC)-modified PAMAM, we synthesized FITC-PAMAM, FITC-PAMAM-PEG and FITC-PAMAMPEG-GE11. Fluorescence microscopy and flow cytometry were used to monitor the uptake of A375 cells of these three nanomaterials. Secondly, TMZ-PAMAM-PEG-GE11-HA drug complexes were prepared by ultrasonic emulsification, and their particle size, zeta potential and morphology were evaluated by DLS and TEM. Drug loading (DL) and encapsulation efficiency (EE) were assayed by ultraviolet spectrophotometry. Thirdly, we ascertained whether TMZ-PAMAM-PEG-GE11-HA conjugates could target A375 cells in vitro. The TMZ-PAMAM-PEG-GE11-HA nanodrug delivery system was successfully synthesized according to FTIR and ${ }^{1} \mathrm{H}-\mathrm{NMR}$. Its mean particle size was $183.2 \mathrm{~nm}$ and zeta potential was $-0.01 \mathrm{mV}$. It was a regular sphere with good uniformity. The EE of TMZ-PAMAM-PEG-GE11-HA was $\sim 50.63 \%$ and DL $\sim 10.4 \%$. TMZ-PAMAM-PEG-
\end{abstract}

Correspondence to: Dr Guan Jiang or Professor Yanqun Liu, Department of Dermatology, Affiliated Hospital of Xuzhou Medical College, 99 West Huai Hai Road, Xuzhou, Jiangsu 221002, P.R. China

E-mail: dr.guanjiang@gmail.com

E-mail: 5721268@163.com

${ }^{*}$ Contributed equally

Key words: melanoma, nanotechnology, PAMAM, temozolomide, GE11, A375 cells
GE11-HA targeted A375 cells in vitro. In conclusion, the TMZ-PAMAM-PEG-GE11-HA nanodrug delivery system was successfully prepared, and demonstrated its potential for targeting A375 cells in vitro. This system enhanced the sensitivity of A375 cells to TMZ, and provided a novel targeted strategy for the treatment of metastatic melanoma.

\section{Introduction}

Malignant melanoma is responsible for $4 \%$ of all skin cancer cases, but accounts for $79 \%$ of skin cancer-related deaths (1). First-line treatment includes resection of the primary tumor. If all cancerous cells are not removed, the risk of metastasis and poor survival is high (2).

In recent years, with the development of targeted immune and individualized targeted therapies, treatment of melanoma has made considerable progress (3). The latest research results using programmed cell death protein (PD)-1 have pushed the treatment of advanced melanoma to new levels. Data show that the anti-PD-1 monoclonal antibodies MK-3475 and BMS-936558 have a mean efficiency of $40 \%$, complete remission rate of $17 \%$, and that progression-free survival is $\sim 2$ years (4). However, the relationship between expression of programmed death-ligand-1 in tumor tissues and the efficacy of single agent PD-1 inhibition is controversial (5). Therefore, chemotherapy for Chinese patients with melanoma remains an important means of treatment.

The alkylating agent temozolomide (TMZ) is recommended in several countries for the first-line treatment of malignant melanoma (6). It is thought that TMZ passes through the blood-brain barrier and has high bioavailability, and could be used to prevent/treat brain metastasis. TMZ causes DNA single-strand or double-strand breaks and blocks DNA replication, leading to the death of tumor cells through methylation of DNA chains (7). In recent years, chemotherapy for malignant melanoma has not made significant progress. The poor prognosis of malignant melanoma is related directly to its high capacity for tissue infiltration and its resistance to chemotherapeutic drugs (8).

Nanotechnology has revolutionized cancer treatment. Attachment of a drug to a carrier enables the fast transfer of the complex to the tumor. In addition, sustained release of 
the drug achieves an effective concentration while reducing systemic toxicity (9).

The polyamide-amine dendrimer (PAMAM) has a hyperbranched, symmetrical and radiating structure $(10,11)$. PAMAM has three obvious structural advantages compared with other carrier molecules $(12,13)$. Firstly, PAMAM has a diameter of 1-15 nm with a wide cavity for embedding of a drug. Secondly, PAMAM is susceptible to chemical modification to better protect the drug from enzymatic degradation. Thirdly, the termini of PAMAM contain several reactive functional groups, which enable ligand binding to target cells. A PAMAM-based drug-carrier system enables gradual accumulation of the drug in tumor tissue based on the enhanced permeability and retention (EPR) effect. Compared with the free drug, the drug concentration in tumor tissue is increased, thereby aiding the passive targeting of tumor cells.

The unique role of the epidermal growth factor receptor (EGFR) in the growth and metastasis of tumors has made it a 'hot topic' in the development of targeted cancer drugs (14). GE11 is a small polypeptide comprising 11 amino acids, and is a ligand of the EGFR. Song et al (15) showed that GE11 modified with liposomes has high targeting efficiency for the EGFR.

Based on previous studies, we used PAMAM as a polymer framework material. We employed NHS-PEG-MAL as a polymer modifier and connecting bridge. The active amino group of PAMAM is bound to one end of the NHS-PEG active carboxyl group via an acidamide reaction, whereas the other end of the PEG active group (-MAL) is connected to the active group (-SH) of the targeting agent (GE11 polypeptide). Then, using hyaluronic acid (HA) as an emulsifier, TMZ is embedded by 'phacoemulsification' to form the composite TMZ-PAMAM-PEG-GE11-HA (Fig. 1). Continued and sustained release of $\mathrm{TMZ}$ enables reduced accumulation of $\mathrm{TMZ}$ in normal tissues, and thus reduces the systemic side-effects of this drug.

\section{Materials and methods}

Chemicals. Generation-5 poly(amidoamine) dendrimer (G5 PAMAM dendrimer) was purchased from Xian Ruixi Biological Technology (Xian, China). MEO-PEG-NHS and MAL-PEG-NHS were obtained from JenKem Technology (Beijing, China). GE11-SH was purchased from ChinaPeptides (Beijing, China). Temozolomide (TMZ) was obtained from TCI Shanghai (Shanghai, China). 4',6-Diamidino-2-phenylindole (DAPI) was purchased from Invitrogen (Carlsbad, CA, USA). $N, N$-dimethylformamide (DMF), dimethyl sulfoxide (DMSO), methanol, acetonitrile, fluorescein isothiocyanate (FITC) and all other chemicals were purchased from Sigma-Aldrich (St. Louis, MO, USA).

Synthesis of PAMAM-PEG. PAMAM and MEO-PEG-NHS (1:48) were added to phosphate-buffered saline (PBS; pH 8.0) and stirred for $12 \mathrm{~h}$ at room temperature. The reaction mixture was dialyzed using a dialysis tube [molecular weight cut-off (MWCO), $15 \mathrm{kDa}$ ) for $24 \mathrm{~h}$. The aqueous solution was lyophilized to afford PAMAM-PEG as a white solid.

Synthesis of PAMAM-PEG-GE11. PAMAM (10 mg) and MEO-PEG-NHS (83.26 mg) were added to PBS (pH 8.0) to synthesize PAMAM-PEG. To a solution of PAMAM-PEG in PBS, GE11-SH (9.04 mg) in acetonitrile was added. After stirring for $3 \mathrm{~h}$ at room temperature, the $\mathrm{pH}$ of the reaction mixture was regulated to 7.0 and $\beta$-mercaptoethanol $(55.5 \mu \mathrm{mol})$ was added. After stirring for $1 \mathrm{~h}$, the reaction mixture was dialyzed using a dialysis tube (MWCO, $15 \mathrm{kDa}$ ) for $24 \mathrm{~h}$. The aqueous solution was lyophilized to afford PAMAM-PEG-GE11 as a white solid.

FITC-labeled dendrimers were synthesized by coupling FITC to the amino group of the proximal lysine group between PEG and cholic acid after removal of the deprotecting group in methanol solution.

Fourier transform-infrared (FTIR) and nuclear magnetic resonance (NMR) spectroscopy. The chemical structures of the three polymers, PAMAM, PAMAM-PEG and PAMAM-PEG-GE11, were identified by analyses of FTIR and proton NMR ( $\left.{ }^{1} \mathrm{H}-\mathrm{NMR}\right)$ spectra. FTIR spectra of these polymers were recorded on a spectrophotometer (Olympus, Tokyo, Japan) using $\mathrm{KBr}$ as a reference. ${ }^{1} \mathrm{H}-\mathrm{NMR}$ spectra of these polymers were obtained using an MR 400 NMR system (Agilent Technologies, Palo Alto, CA, USA), with samples dissolved in deuterium oxide $\left(\mathrm{D}_{2} \mathrm{O}\right)$ or DMSO, respectively.

Characterization of PAMAM-PEG-GE11. The mean size, polydispersity index and zeta potential of PAMAM-PEG-GE11 were determined by dynamic light scattering (DLS) using a ZetaSizer (Nano ZS90; Malvern Instruments, Malvern, UK). Determinations were made when samples were diluted in distilled water $(\sim 1 \mathrm{mg} / \mathrm{ml})$. Morphology of the samples was characterized by transmission electron microscopy (TEM) using Tecnai ${ }^{\mathrm{TM}}$ G2 F20 U-TWIN (FEI, Eindhoven, The Netherlands).

Cell culture. Human melanoma cells (A375) and human skin fibroblasts (HSFs) were purchased from Shanghai Cell Collection (Shanghai, China). Cells were grown in an atmosphere of $5 \% \mathrm{CO}_{2}$ and relative humidity of $95 \%$ using DMEM (Gibco-BRL, Grand Island, NY, USA) supplemented with $10 \%$ fetal bovine serum and $1 \%$ penicillin-streptomycin solution.

Cell uptake. Cell-uptake studies were undertaken to investigate whether three nanomaterials, FITC-PAMAM, FITC-PAMAM-PEG and FITC-PAMAM-PEG-GE11, were internalized by receptor-mediated endocytosis. Briefly, A375 cells $\left(4 \times 10^{4} /\right.$ well) were seeded into 24 -well plates overnight before experimentation. Cells were incubated with $5 \mathrm{nM}$ of these three nanomaterials at $37^{\circ} \mathrm{C}$ for $4 \mathrm{~h}$. The cells were washed thrice with PBS ( $\mathrm{pH} 7.4$ ), fixed with $4 \%$ paraformaldehyde and stained with DAPI for $5 \mathrm{~min}$. Then, the cells were washed thrice with PBS. Fluorescence micrographs of cells were captured using an inverted microscope (IX70; Olympus).

A375 cells ( $2 \times 10^{5} /$ well) were seeded in 6 -well plates overnight before experimentation. Cells were incubated with $5 \mathrm{nM}$ of these three nanomaterials at $37^{\circ} \mathrm{C}$ for $4 \mathrm{~h}$. Cells were washed thrice with PBS ( $\mathrm{pH} 7.4$ ), trypsinized and centrifuged at $0.4 \mathrm{x} \mathrm{g}$ for $5 \mathrm{~min}$ at room temperature. After washing twice with PBS, the samples underwent flow cytometry (FACSCalibur ${ }^{\mathrm{TM}}$ flow cytometer; BD Biosciences, Franklin Lakes, NJ, USA). 
Preparation and characterization of TMZ-PAMAM-PEGGE11-HA complexes. TMZ-PAMAM-PEG-GE11-HA complexes were prepared by ultrasonic emulsification. Briefly, TMZ (1 mg) and PAMAM-PEG copolymers (10 mg) were dissolved in methanol $(1 \mathrm{ml})$. In addition, TMZ (3 mg) and HA (10 mg) were dissolved in $4 \mathrm{ml}$ aqueous solution. The solution of TMZ and PAMAM-PEG copolymers was added drop-by-drop to the aqueous solution with vigorous stirring at room temperature. Then, the mixture was emulsified by sonication for $10 \mathrm{~min}$ at $40 \mathrm{~W}$, and evaporated under reduced pressure to remove the remaining methanol. These complexes were transferred to an ultrafiltration tube (MWCO, $10 \mathrm{kDa})$, centrifuged at $3.5 \mathrm{x} \mathrm{g}$ for $10 \mathrm{~min}$ at room temperature, and washed with deionized water thrice. DLS and TEM were undertaken to evaluate the particle size, zeta potential and morphology of the TMZ-PAMAM-PEG-GE11-HA complexes.

Loading rate $(L R)$ and encapsulation efficiency $(E E)$ of the TMZ-PAMAM-PEG-GE11-HA complexes. The LR and EE were assayed by ultraviolet spectrophotometry. A certain amount of TMZ was dissolved in DMF (to $10 \mu \mathrm{g} / \mathrm{ml}$ ) and its absorption peak was measured by an ultraviolet spectrophotometer with DMF as a blank. Standard solutions of TMZ $(4,6,8,10$ and $16 \mu \mathrm{g} / \mathrm{ml})$ were prepared. The absorbance of different concentrations was measured with UV detection at $329 \mathrm{~nm}$ and a linear regression equation was obtained. An ultrafiltration tube (MWCO, $10 \mathrm{kDa}$ ) was used to separate free TMZ. LR and EE were defined as follows:

$$
\begin{aligned}
& \mathrm{LR}=\left(\mathrm{W}_{0}-\mathrm{W}_{\mathrm{f}}\right) / \mathrm{W}_{0} \times 100 \% \\
& \mathrm{EE}=\left(\mathrm{W}_{0}-\mathrm{W}_{\mathrm{f}}\right) / \mathrm{W}_{\mathrm{s}} \times 100 \%
\end{aligned}
$$

where $\mathrm{W}_{0}$ and $\mathrm{W}_{\mathrm{f}}$ are the weight of initial TMZ and free $\mathrm{TMZ}$ detected in solution, respectively, and $\mathrm{W}_{\mathrm{s}}$ is the weight of the complex after lyophilization.

Statistical analyses. Statistical analyses were conducted using the Student's t-test for comparison of two groups and one-way ANOVA for multiple groups. $\mathrm{P}<0.05$ was considered to indicate a statistically significant result.

\section{Results}

Synthesis of PAMAM-PEG and PAMAM-PEG-GE11 compounds. G5 PAMAM dendrimer with 128 amino groups was used in the present study. To reduce their cytotoxicity and increase their circulation and targeting of cancer cells, PEG and GE11 polypeptide were modified on the surface of the dendrimer (Fig. 1).

FTIR spectra showed characteristic peaks of the hydroxyl and ether groups of PEG at 3,437 and 1,109 $\mathrm{cm}^{-1}$, and those of amide and carbonyl groups peak were found at 3,286 and $1,647 \mathrm{~cm}^{-1}$. These results successful confirmed coupling of PEG and PAMAM. We did not find the typical peak of GE11 in the FTIR spectra of PAMAM-PEG-GE11, probably due to duplication at $1,104.9 \mathrm{~cm}^{-1}$ of the stretching vibration peak of C-O-C (das) in PAMAM-PEG and hydroxyl group $(v \mathrm{OH})$ in GE11 (Fig. 2A). NMR was used to confirm the success of coupling of PEG and PAMAM. Protons of PAMAM are derived from the $-\mathrm{CH}_{2}-\mathrm{CH}_{2}-\mathrm{N}$ - structure and their chemical shift is 2.2-3.4 ppm. Protons in PEG have a chemical shift at $3.4-3.8 \mathrm{ppm}$, and the characteristic methylene peak of PEG was found at $3.60 \mathrm{ppm}$, and the characteristic peak of the methyl groups at $3.42 \mathrm{ppm}$. The characteristic peak of PEG and PAMAM could be found in the ${ }^{1} \mathrm{H}-\mathrm{NMR}$ spectrum of PEG-PAMAM in $\mathrm{D}_{2} \mathrm{O}$, suggesting that PEG-PAMAM was successfully synthesized (Fig. 2B and C). Protons in the GE11 polypeptide have a chemical shift at $6.5-9.3 \mathrm{ppm}$. The ${ }^{1} \mathrm{H}-\mathrm{NMR}$ spectrum of PAMAM-PEG-GE11 in DMSO displayed characteristic peaks of the PAMAM-PEG and GE11 polypeptide (Fig. 2D-F). Taken together, these results suggest that the nanodrug delivery system was successfully created.

Characterization of the PAMAM-PEG-GE11 conjugates. TEM and DLS were carried out to characterize the PAMAMPEG-GE11 conjugates. Transmission electronic micrographs showed that the PAMAM-PEG-GE11 conjugates were close to spherical and that the mean diameter was $40 \mathrm{~nm}$ (Fig. 3). The mean hydrodynamic diameter measured by DLS was $249.2 \pm 18.4 \mathrm{~nm}$, which may have been due to hydration shells. The zeta potential of PAMAM-PEG-GE11 conjugates was $20.2 \pm 0.8 \mathrm{mV}$. The reduced positive charge compared with that of PAMAM $(\sim 40 \mathrm{mV})$ may have been due to the fact that the terminal amino groups of PAMAM were partly neutralized.

Active targeting of PAMAM-PEG-GE11 to A375 cells. To assess active targeting of PAMAM-PEG-GE11 conjugates to A375 cells (which overexpress the EGFR), fluorescence microscopy and flow cytometry were carried out. Three nanomaterials, PAMAM, PAMAM-PEG and PAMAM-PEG-GE11, were modified with FITC. Fluorescence microscopy images showed PAMAM (Fig. 4A), PAMAM-PEG (Fig. 4B) and PAMAM-PEG-GE11 (Fig. 4C) to be present in different amounts in the A375 cells (blue, cell nucleus; green, FITC-nanomaterials). This result showed that all three nanomaterials entered the A375 cells. In addition, coupling with GE11 was internalized considerably more than with PAMAM and PAMAM-PEG. Notably, the amount of nanocarriers that entered the cells was slightly reduced whether PAMAM was modified with PEG. The reason for this phenomenon may be neutralization of the positive charge on the PAMAM surface by PEG, which may result in reduction in electrostatic forces between PAMAM and cell membranes. Subsequently, we studied the fluorescence intensity of PAMAM, PAMAM-PEG and PAMAM-PEG-GE11 in the A375 cells by flow cytometry, which elicited results that were in accordance with those of the fluorescence microscopy (Fig. 4D). Taken together, these results showed that PAMAM-PEG-GE11 could be transported to A375 cells in vitro.

Measurement of LR and EE of the TMZ-PAMAM-PEGGE11-HA conjugates. TMZ was dissolved in DMF, and a concentration of $10 \mu \mathrm{g} / \mathrm{ml}$ was measured by ultraviolet-visible spectrometry. The absorption spectrum showed that the typical intense absorption peak of TMZ was at $329 \mathrm{~nm}$ (Fig. 5A), which could be used to quantify the TMZ concentration. To measure the EE and LR of the TMZ-PAMAM-PEG-GE11-HA conjugates, the absorbance of different concentrations of TMZ was measured (Fig. 5B), and a linear regression equation is 


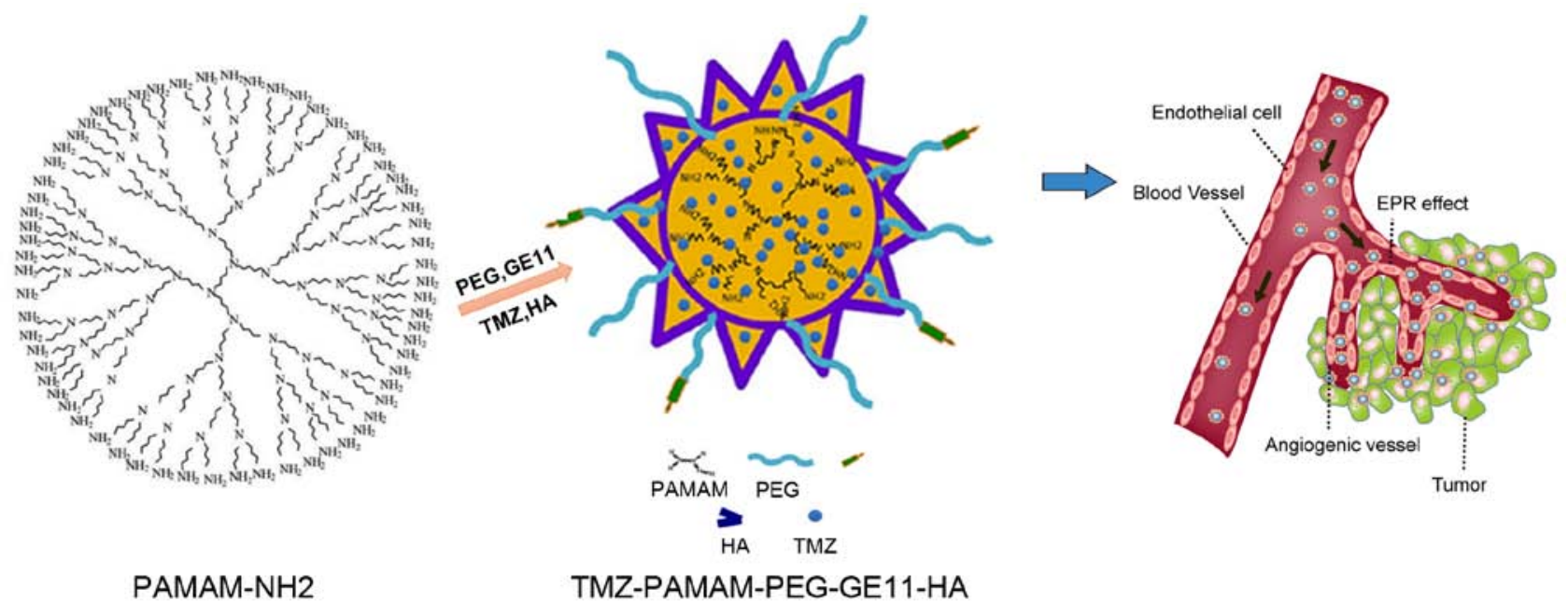

Figure 1.Chemical structure of the G5 PAMAM dendrimer with 128 amino groups and preparation of the targeted TMZ-loaded nanoparticles. Alkyne-containing GE11 polypeptide was conjugated to the azide group on the distal terminus of a PEG chain. GE11 polypeptides on the surface of the targeted nanoparticles can recognize and bind the EGFR (which is overexpressed on the cell membrane of melanoma cells) specifically.

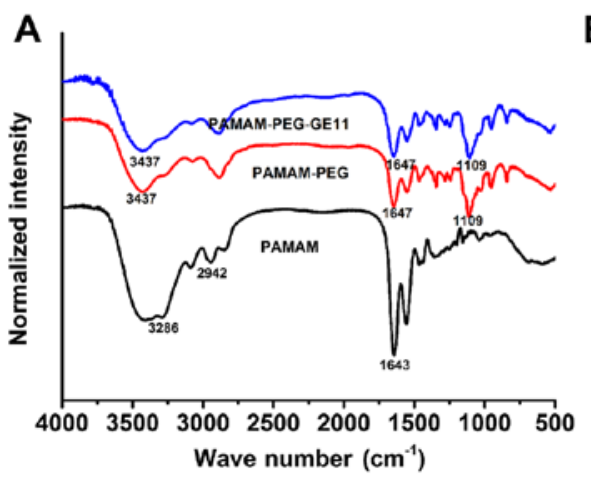

D

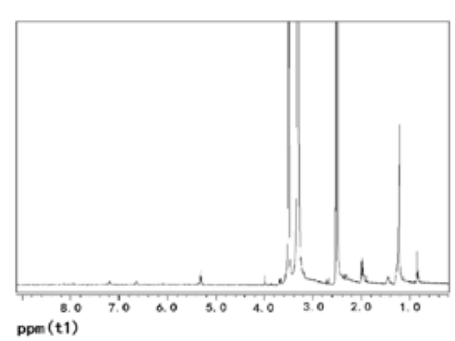

B

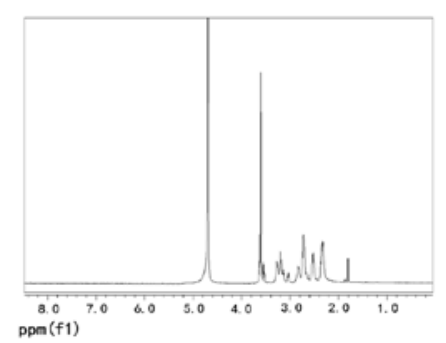

E

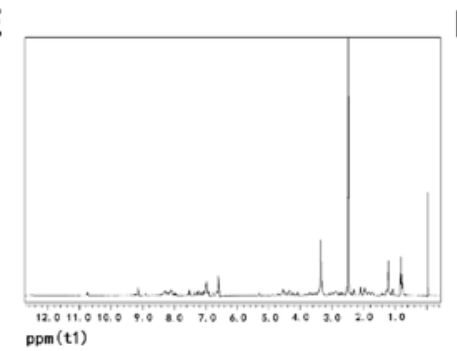

C

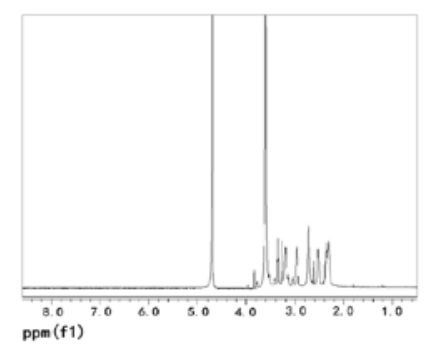

$\mathbf{F}$

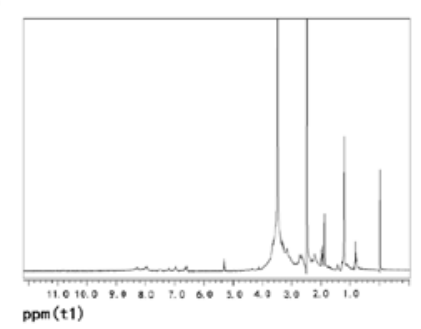

Figure 2. (A) FTIR and (B-F) ' $\mathrm{H}-\mathrm{NMR}$ of PAMAM, PAMAM-PEG and PAMAM-PEG-GE11 with $\mathrm{D}_{2} \mathrm{O}$ of DMSO as solvent. ${ }^{1} \mathrm{H}-\mathrm{NMR}$ spectra of (B) PAMAM, (C and D) PAMAM-PEG, (E) GE11 and (F) PAMAM-PEG-GE11.

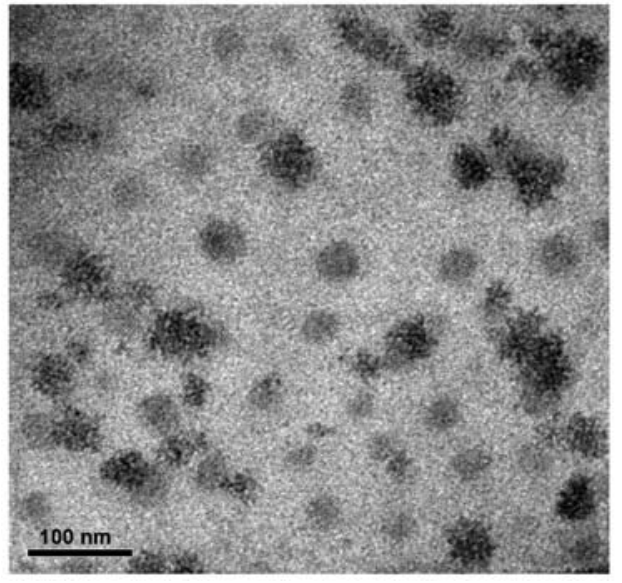

provided (absorbance $=0.0495 \mathrm{x}$ concentration +0.0256 ; $\mathrm{r}=0.9999)$. The EE and LR of the synthesized TMZ-PAMAMPEG-GE11-HA conjugates were 50.63 and $10.40 \%$, respectively.

Characterization of the TMZ-PAMAM-PEG-GE11-HA conjugates. To characterize the TMZ-PAMAM-PEGGE11-HA conjugates, DLS was carried out to measure the hydrodynamic size and zeta potential. The mean hydrodynamic diameter was $183.2 \mathrm{~nm}$ (Fig. 6A). The zeta potential of the TMZ-PAMAM-PEG-GE11-HA conjugates was $-0.01 \mathrm{mV}$ (approximate to neutral), and the zeta deviation was $5.91 \mathrm{mV}$, 

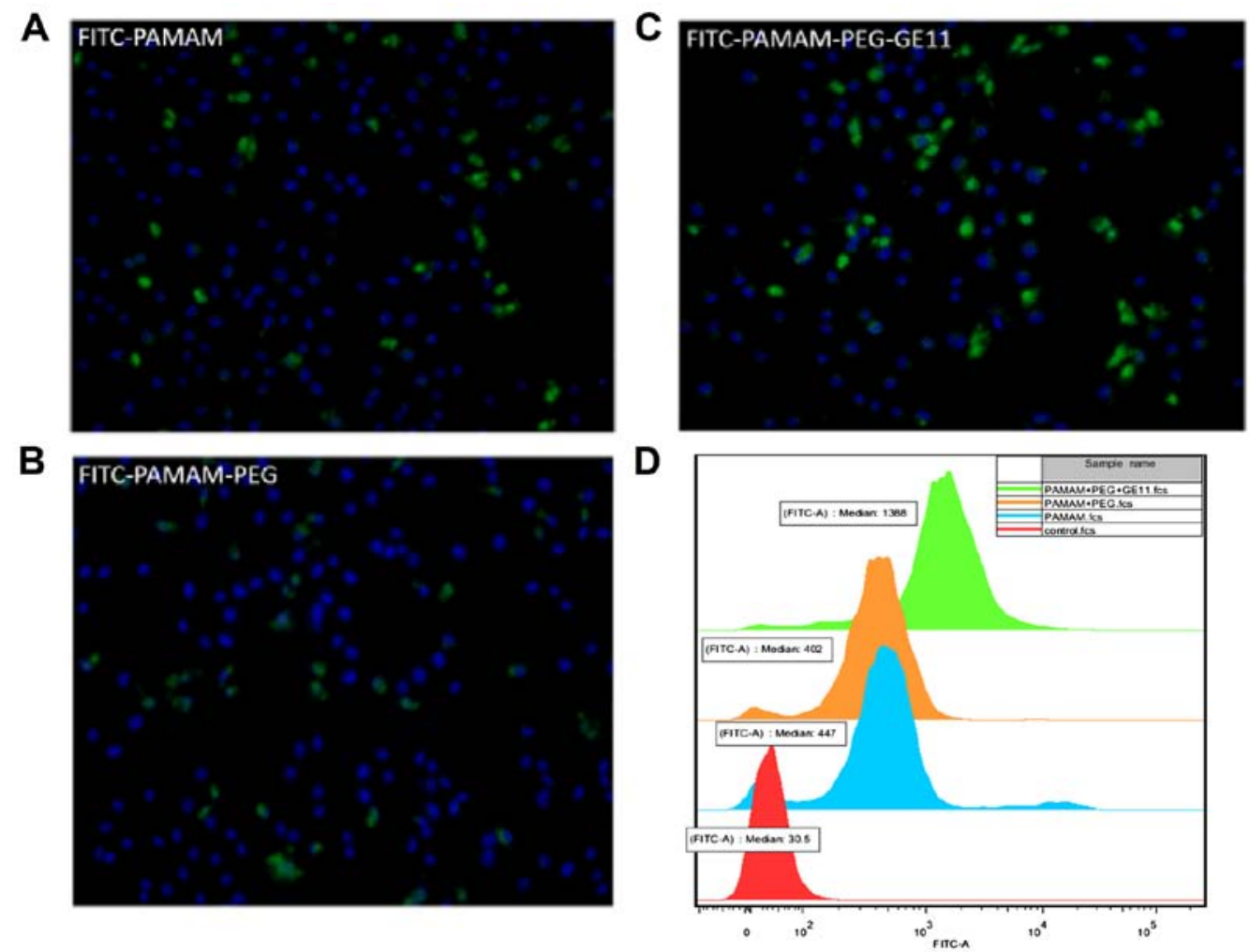

Figure 4. Cell-uptake studies in A375 cells in vitro. (A-C) Fluorescence micrographs (magnification, x100) and (D) flow cytometry of FITC-labeled PAMAM, PAMAM-PEG and PAMAM-PEG-GE11.

A

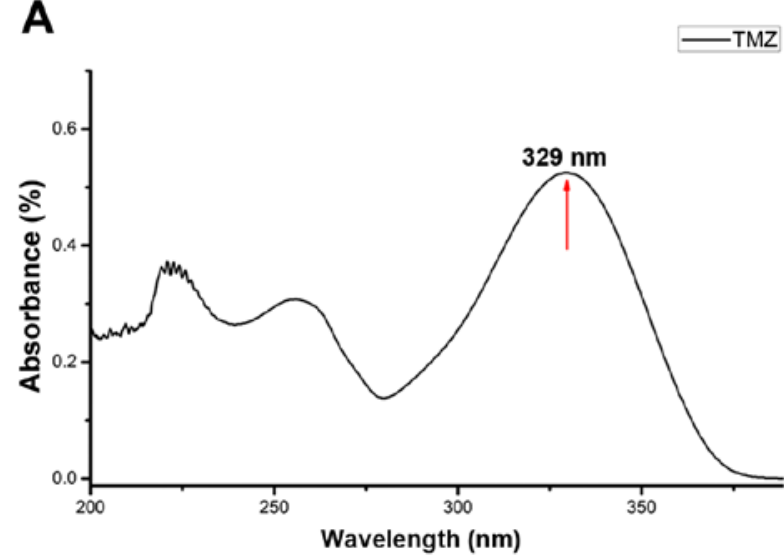

B

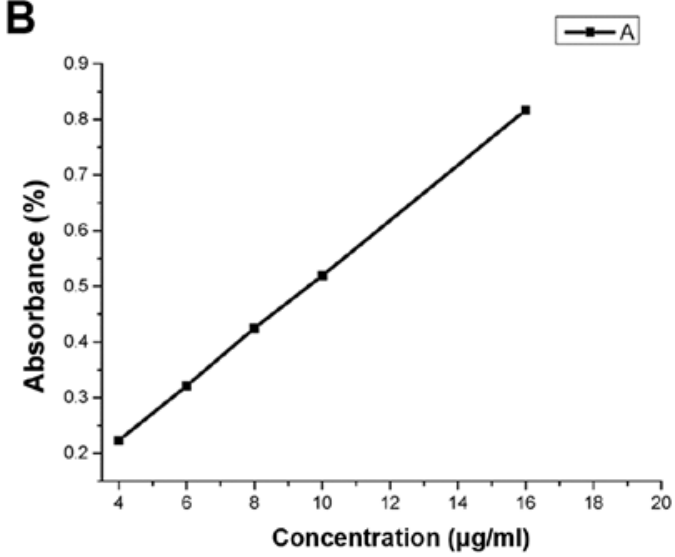

Figure 5. (A) Absorption peak of TMZ with DMF as solvent and at a concentration of $10 \mu \mathrm{g} / \mathrm{ml}$. (B) Linear standard curve of absorbance (A) to different concentrations of TMZ standard solution measured using UV detection at $329 \mathrm{~nm}$.

suggesting good dispersion in the dissolution and low toxicity (Fig. 6B). Transmission electron micrographs showed that the TMZ-PAMAM-PEG-GE11-HA conjugates were close to spherical and that their size was $\sim 183.2 \mathrm{~nm}$ (Fig. 6C).

Uptake of the TMZ-PAMAM-PEG-GE11-HA conjugates. Uptake of the TMZ-PAMAM-PEG-GE11-HA conjugates by HSFs and A375 cells was analyzed by fluorescence microscopy. After treatment with the TMZ-PAMAM-PEG-GE11-HA conjugates for $4 \mathrm{~h}$, samples were fixed and stained. The fluorescence intensity of the conjugates in the A375 cells was stronger than that noted in the HSFs (Fig. 7). This result suggested that TMZ-loading nanocarriers modified by GE11 entered malignant melanoma cells efficiently, and that only a negligible amount were internalized by normal tissue cells.

\section{Discussion}

Melanoma is one of the most malignant forms of skin cancer. Early-stage melanoma is curable by surgery, but if it becomes widespread, the survival rate is extremely low owing to its poor response to drugs (16). Conventional treatments (e.g., surgery, chemotherapy and radiotherapy) combined with immunotherapy and molecular-targeted therapy, have improved the 
A
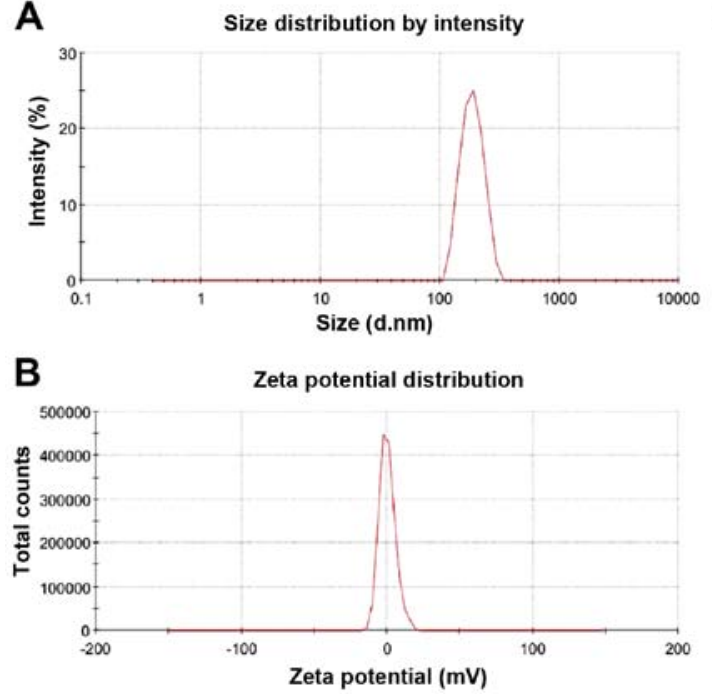

C

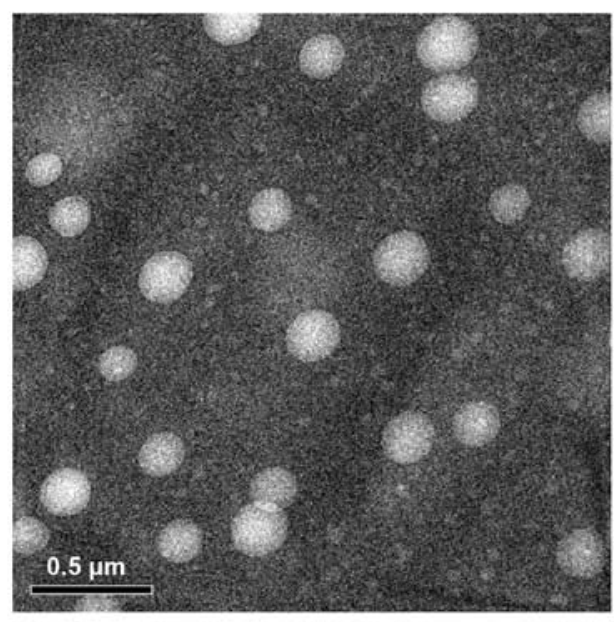

Figure 6. Characterization of TMZ-PAMAM-PEG-GE11-HA. (A and B) Size distribution and zeta potential of TMZ-PAMAM-PEG-GE11-HA in ddH ${ }_{2} \mathrm{O}$ at $1 \mathrm{mg} / \mathrm{ml}$. (C) Transmission electron micrograph of TMZ-PAMAM-PEG-GE11-HA.

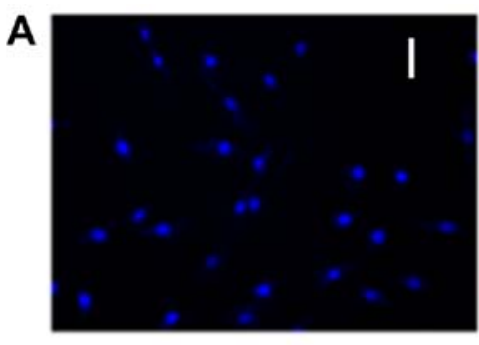

B

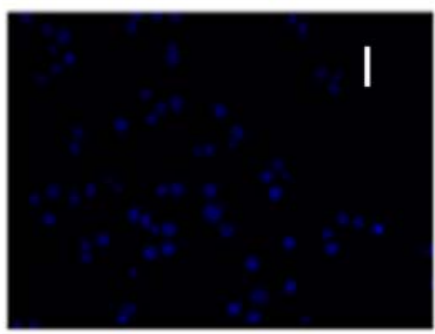

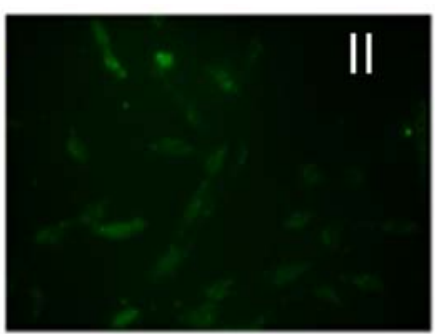
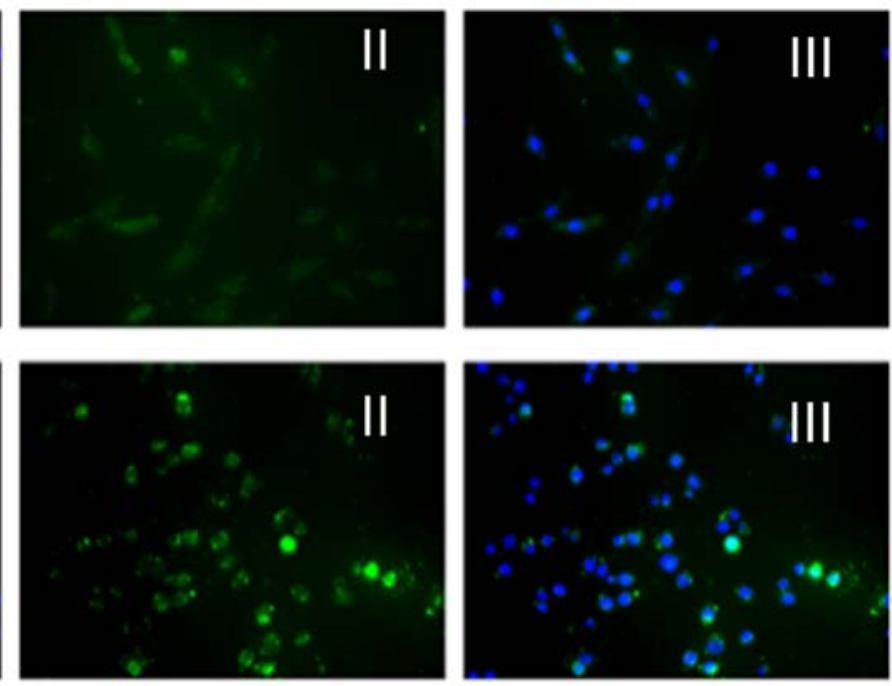

Figure 7. Uptake of FITC-TMZ-PAMAM-PEG-GE11-HA complexes by (A) HSFs and (B) A375 cells. I, nucleus; II, FITC-TMZ-PAMAM-PEG-GE11-HA; III, merge (I and II) (magnification, $x 400$ ).

prognosis of patients with malignant melanoma (17). However, radiotherapy and chemotherapy can kill cancer cells, but also normal cells, thus, development of more effective delivery systems to target tumor cells is particularly urgent.

TMZ has poor solubility and stability in water. Whether administered via the oral route, its half-life is $1.8 \mathrm{~h}$, and it is rapidly cleared from the circulation (18). Nanocarriers can increase the water solubility of TMZ, aid sustained release of the drug, and thus, improve the half-life of TMZ under physiologic conditions, which protects it from degradation and loss of therapeutic effects (19).

In the present study, PAMAM dendrimers were modified with PEG by chemical synthesis. Due to the hydrophilicity and flexibility of PEG, PEG-modified compounds lower the risk of being recognized by macrophages after intravenous injection, thus, they cannot be phagocytized readily by macrophages. Therefore, compared with conventional nanodrug delivery systems and the original drug, PEG-modified compounds can extend the plasma half-life of TMZ. Hence, targeted tumor therapy through enhancement of the EPR effect can be carried out.

Thomas et al (20) suggest that macromolecular drugs of molecular weight $>35 \mathrm{kDa}$ and drug systems of particle size $<200 \mathrm{~nm}$ can take advantage of the EPR effect whether targeted at tumor tissue. The TMZ-PAMAM-PEG-GE11-HA nanoparticles prepared in the present study (molecular weight $>35 \mathrm{kDa}$; mean particle diameter $=183.2 \mathrm{~nm}$ ) suggest that the EPR effect can be enhanced. However, a particle size for TMZ-PAMAM-PEG-GE11-HA conjugates of $183.2 \mathrm{~nm}$ also reduces cell uptake. Usually, different sizes of nanoparticles can enter cells by different routes, including directly through the membrane and by endocytosis $(21,22)$, and complexes with a particle size of 10-50 $\mathrm{nm}$ can enter cells more efficiently (23). However, we created a new active targeting complex: 
TMZ-PAMAM-PEG-GE11-HA. In addition to undergoing non-specific uptake by melanoma cells, they can also be taken up through ligand receptor-mediated endocytosis in a cell-specific way.

The GE11 polypeptide is a ligand of the EGFR. The latter is highly expressed on the surface of many types of tumor cells (including melanoma cells) and is closely related with tumor development and prognosis. Hence, by targeting the EGFR, a GE11 polypeptide-modified delivery system can identify and locate the tumor site specifically, and be rapidly taken up by A375 cells. We found that the GE11 polypeptidemodified nanocarrier PAMAM-PEG-GE11 was taken up by cells to a greater extent than that observed for PAMAM and PAMAM-PEG. In addition, PAMAM-PEG-GE11 was covered with a layer of HA to aid drug loading. As an emulsifier, HA enables PAMAM to embed a greater amount of the drug and neutralize the positive charge on the PAMAM surface, thereby reducing the cytotoxicity and hemolytic toxicity of the cationic polymer. HA also exerts two-way targeting with the GE11 polypeptide through binding to CD44 molecules (which are highly expressed on the surface of melanoma cells) (24). Systemic side-effects can be reduced by targeting drug delivery to the tumor site and reducing drug accumulation in normal tissues.

Various studies have shown that HA-modified liposomes bind to the melanoma cell line B16-F10 more readily (and with higher expression of CD44 receptors) than the fibroblast cell line CV-1 (which does not have CD44 receptors) $(24,25)$. Similarly, the presents study showed that binding of A375 cells to nanomaterials was greater than that observed for HSFs. This phenomenon can be explained by the fact that the membrane surface of A375 cells expresses many CD44 molecules, whereas low expression of CD44 molecules is observed in normal cells. Thus, the HA-modified drug complex TMZ-PAMAM-PEGGE11-HA can bind to A375 cells more readily. Therefore, we showed that the TMZ-PAMAM-PEG-GE11-HA conjugates can target melanoma cells in vitro. However, whether the TMZ-PAMAM-PEG-GE11-HA conjugates can target melanoma cells in vivo may require animal experiments. In addition, $\mathrm{TMZ}$ is unstable in phosphate buffer $(\mathrm{pH}=6.8$ and $\mathrm{pH}=5.5)$, and is broken down readily to the intermediate 3-methyl-(triazen1-yl)imidazole-4-carboximide (MTIC). However, it has been reported that MTIC is relatively stable (26); thus, the TMZ content is indirectly measured. However, in the present study, MTIC was unstable in a shaker at $37^{\circ} \mathrm{C}$, thus, detection of TMZ release was difficult. Therefore, release of the drug TMZ in TMZ-PAMAM-PEG-GE11-HA conjugates remains unclear.

In conclusion, the TMZ-PAMAM-PEG-GE11-HA nanodrug delivery system was successfully prepared, and its potential for targeting human melanoma (A375) cells in vitro was demonstrated. This system enhanced the sensitivity of A375 cells to TMZ, and provides a novel targeted strategy for the treatment of metastatic melanoma.

\section{References}

1. Jemal A, Siegel R, Ward E, Murray T, Xu J and Thun MJ: Cancer statistics, 2007. CA Cancer J Clin 57: 43-66, 2007.

2. Das Thakur M, Salangsang F, Landman AS, Sellers WR, Pryer NK, Levesque MP, Dummer R, McMahon M and Stuart DD: Modelling vemurafenib resistance in melanoma reveals a strategy to forestall drug resistance. Nature 494: $251-255,2013$
3. Luke JJ and Schwartz GK: Chemotherapy in the management of advanced cutaneous malignant melanoma. Clin Dermatol 31: 290-297, 2013

4. Nakagawa E, Aimi Y, Yasuhara O, Tooyama I, Shimada M, McGeer PL and Kimura H: Enhancement of progenitor cell division in the dentate gyrus triggered by initial limbic seizures in rat models of epilepsy. Epilepsia 41: 10-18, 2000.

5. Luke JJ and Ott PA: PD-1 pathway inhibitors: The next generation of immunotherapy for advanced melanoma. Oncotarget 6: 3479-3492, 2015.

6. Biasco G, Pantaleo MA and Casadei S: Treatment of brain metastases of malignant melanoma with temozolomide. N Engl J Med 345: 621-622, 2001.

7. Johannessen TC and Bjerkvig R: Molecular mechanisms of temozolomide resistance in glioblastoma multiforme. Expert Rev Anticancer Ther 12: 635-642, 2012.

8. Ott PA, Chang J, Madden K, Kannan R, Muren C, Escano C, Cheng X, Shao Y, Mendoza S, Gandhi A, et al: Oblimersen in combination with temozolomide and albumin-bound paclitaxel in patients with advanced melanoma: A phase I trial. Cancer Chemother Pharmacol 71: 183-191, 2013.

9. Bourzac K: Nanotechnology: Carrying drugs. Nature 491: S58-S60, 2012.

10. Dufès C, Uchegbu IF and Schätzlein AG: Dendrimers in gene delivery. Adv Drug Deliv Rev 57: 2177-2202, 2005.

11. Singh I, Rehni AK, Kalra R, Joshi G and Kumar M: Dendrimers and their pharmaceutical applications - a review. Pharmazie 63: 491-496, 2008

12. Svenson S: Dendrimers as versatile platform in drug delivery applications. Eur J Pharm Biopharm 71: 445-462, 2009.

13. Sadekar S and Ghandehari H: Transepithelial transport and toxicity of PAMAM dendrimers: Implications for oral drug delivery. Adv Drug Deliv Rev 64: 571-588, 2012.

14. Vetter A, Virdi KS, Espenlaub S, Rödl W, Wagner E, Holm PS, Scheu C, Kreppel F, Spitzweg C and Ogris M: Adenoviral vectors coated with PAMAM dendrimer conjugates allow CAR independent virus uptake and targeting to the EGF receptor. Mol Pharm 10: 606-618, 2013.

15. Song S, Liu D, Peng J, Sun Y, Li Z, Gu JR and Xu Y: Peptide ligand-mediated liposome distribution and targeting to EGFR expressing tumor in vivo. Int J Pharm 363: 155-161, 2008.

16. Rubin KM: Management of primary cutaneous and metastatic melanoma. Semin Oncol Nurs 29: 195-205, 2013.

17. Maverakis E, Cornelius LA, Bowen GM, Phan T, Patel FB, Fitzmaurice S, He Y, Burrall B, Duong C, Kloxin AM, et al: Metastatic melanoma - a review of current and future treatment options. Acta Derm Venereol 95: 516-524, 2015.

18. del Burgo LS, Hernández RM, Orive G and Pedraz JL: Nanotherapeutic approaches for brain cancer management. Nanomedicine 10: 905-919, 2014.

19. Patil R, Portilla-Arias J, Ding H, Inoue S, Konda B, Hu J, Wawrowsky KA, Shin PK, Black KL, Holler E, et al: Temozolomide delivery to tumor cells by a multifunctional nano vehicle based on poly( $\beta$-L-malic acid). Pharm Res 27: 2317-2329, 2010.

20. Thomas TP, Majoros IJ, Kotlyar A, Kukowska-Latallo JF, Bielinska A, Myc A and Baker JR Jr: Targeting and inhibition of cell growth by an engineered dendritic nanodevice. J Med Chem 48: 3729-3735, 2005.

21. Xia T, Kovochich M, Liong M,Zink JI and Nel AE: Cationic polystyrene nanosphere toxicity depends on cell-specific endocytic and mitochondrial injury pathways. ACS Nano 2: 85-96, 2008.

22. Conner SD and Schmid SL: Regulated portals of entry into the cell. Nature 422: 37-44, 2003.

23. Albanese A, Tang PS and Chan WC: The effect of nanoparticle size, shape, and surface chemistry on biological systems. Annu Rev Biomed Eng 14: 1-16, 2012.

24. Yoon HY, Koo H, Choi KY, Lee SJ, Kim K, Kwon IC, Leary JF, Park K, Yuk SH, Park JH, et al: Tumor-targeting hyaluronic acid nanoparticles for photodynamic imaging and therapy. Biomaterials 33: 3980-3989, 2012.

25. Eliaz RE and Szoka FC Jr: Liposome-encapsulated doxorubicin targeted to CD44: A strategy to kill CD44-overexpressing tumor cells. Cancer Res 61: 2592-2601, 2001.

26. Appel EA, Rowland MJ, Loh XJ, Heywood RM, Watts C and Scherman OA: Enhanced stability and activity of temozolomide in primary glioblastoma multiforme cells with cucurbit $[n]$ uril. Chem Commun 48: 9843-9845, 2012. 